\title{
ЭКСПЕРИМЕНТАЛЬНОЕ ИССЛЕДОВАНИЕ ТЕПЛОВОГО ГАЗОДИНАМИЧЕСКОГО МЕТОДА ДЛЯ ПРЕДОТВРАЩЕНИЯ ГИДРАТООБРАЗОВАНИЯ В ГАЗОПРОВОДАХ С ИСПОЛЬЗОВАНИЕМ ЦИКЛОНОВ
}

\author{
Зятиков Павел Николаевич', \\ zpavel@tpu.ru
}

\author{
Волков Павел Валерьевич2,1, \\ tpuipr@mail.ru
}

\author{
Большунов Артем Владимирович1, \\ tpuiprgrnm@mail.ru \\ 1 Национальный исследовательский Томский политехнический университет, \\ Россия, 634050, г. Томск, пр. Ленина, 30. \\ 2 АО ТомскНИПИнефть, \\ Россия, 634027, г. Томск, пр. Мира, 72.
}

\begin{abstract}
Актуальность исследования обусловлена необходимостью уменьшения количества гидратов газа в потоке смеси или их полного разрушения. Газовые гидраты являются крайне негативным фрактором газовой промышленности, они усложняют все стадии подготовки, транспортировки и переработки сырья. Гидраты аккумулируются на стенках сосудов при определенных термобарических условиях, также их скопление наблюдается в полостях арматуры, что может привести к их преждевременному износу или повышению гидравлического сопротивления потока флюида, а следовательно, повышению энергических затрат. Существующие методы борьбы с гидратообразованием очень опасны (применение метанола для разрушения гидратов), очень затратны (применение ингибиторов гидратообразования) или энергозатратны и малоэфффективны (прогрев трубопровода специализированным кабелем). Также отложения гидратов могут негативно сказываться на приборах учета и замера.

Цель: экспериментальное исследование процесса удаления из потока газа льда и его разрушения на специализированной установке.

Объект: автономная модульная установка для борьбы с гидратообазованием.

Методы: теоретические методы для обоснования закономерности течения процессов тепломассообмена, расчетноаналитический метод для определения количества энергии, практические методы по проведению экспериментов.

Результаты. Построен лабораторный стенд на базе Национального исследовательского Томского политехнического университета, проведены эксперименты для различных потоков газа с разными термобарическими условиями, проанализированы основные зависимости качества отделения льда из смеси, доказана практическая значимость разработки и даны рекомендации для создания опытного образца. Рассмотрена модель расчета основных конструктивных параметров циклона, рабочей полости. Представлена методика проведения экспериментов и техники подбора оптимальных термобарических параметров.
\end{abstract}

\section{Ключевые слова:}

Гидратообразование, циклон, газодинамический метод, тепломассообмен, динамика.

\section{Введение}

В девятнадцатом столетии гидраты были очень плохо изучены. Цель исследований была посвящена определению химических соединений, которые способны образовывать гидраты, и термобарических параметров, которые необходимы для прохождения реакции [1]. В этот период времени было открыто большое количество гидратообразующих веществ. Только в двадцатом веке газовые гидраты были точно определены и указали на свою важность в промышленной отрасли [2-4].

В газовой промышленности гидраты служат крайне негативным фатором. Их присутствие в потоке смеси замедляет добычу флюида, его транспортировку и сильно усложняет подготовку конечного продукта [5]. При определенных параметрах газовые гидраты аккумулируются на стенках и полостях сосудов, что дает дополнительное гидравлическое сопротивление и уменьшает их срок службы. Присутствие гидратов на установке комплексной подготовки газа и конденсата (УКПГ) негативно сказывается на технологическом процессе и режиме подготовки, что влечет за собой снижение качества конечного продукта [6-9].

Для эффективной борьбы с отложениями гидратов необходимо комплексно учитывать все параметры и условия их образования и диссоциации [10-12]. Система расчета должна быть адаптивной, потому что величина температуры, давления, расхода и компонентного состава непостоянны $[13,14]$. Для решения всех практических задач необходимо иметь детальную физико-математическую модель, учитывающую условия эксплуатации и разработки $[15,16]$.

Объектом исследования является автономная модульная установка, на которой проводились экспериментs по отделению льда.

Исследования выполнены на базе Томского политехнического университета путем проведения более 500 экспериментов с различными термобарическими параметрами. Результаты и выводы представлены в виде графиков и аналитического обзора. 
Расчет физико-математической модели возможного образования гидратов на участке трубопровода

Для построения физической модели применения установки необходимо рассчитать вероятность образования гидратов на участке. Для этого вносятся основные данные от нефтегазодобывающей компании или компании по транспортировке углеводородного сырья (табл. 1, 2) [17-19].

Таблица 1. Начальный компонентный состав газа

Table 1. Initial component composition of gas

\begin{tabular}{|c|c|c|c|c|c|c|c|}
\hline $\begin{array}{c}\text { Компонент } \\
\text { Component }\end{array}$ & $\mathrm{CH}_{4}$ & $\mathrm{C}_{2} \mathrm{H}_{6}$ & $\mathrm{C}_{3} \mathrm{H}_{8}$ & $\mathrm{C}_{4} \mathrm{H}_{10}$ & $\mathrm{C}_{5} \mathrm{H}_{10}$ & $\mathrm{CO}_{2}$ & $\mathrm{~N}_{2}$ \\
\hline $\begin{array}{c}\text { Доля, \% } \\
\text { Part, \% }\end{array}$ & 84,52 & 5,82 & 5,03 & 1,94 & 0,57 & 1,47 & 0,65 \\
\hline
\end{tabular}

Таблица 2. Начальные параметры смеси газа и параметры трубопровода

Table 2. Initial parameters of gas mixture and parameters of pipeline

\begin{tabular}{|c|c|}
\hline Параметр/Parameter & $\begin{array}{l}\text { Значение } \\
\text { Value }\end{array}$ \\
\hline $\begin{array}{l}\text { Длинна участка трубопровода, км } \\
\text { Pipeline length, km }\end{array}$ & $\ell_{K C}=141,25$ \\
\hline $\begin{array}{l}\text { Давление смеси на входе в трубопровод, давление } \\
\text { на выходе, МПа } \\
\text { Fluid pressure at the entrance to the pipeline, } \\
\text { the pressure at the outlet of the system, MPa }\end{array}$ & $P_{H}=6,5 \mathrm{P}_{K}=3$ \\
\hline $\begin{array}{l}\text { Справочное значение теплоемкости газа, Дж/(кг } \cdot \mathrm{K}) \\
\text { Reference value of gas heat capacity, } \mathrm{J} /(\mathrm{kg} \cdot \mathrm{K})\end{array}$ & $C_{P}=2816,46$ \\
\hline $\begin{array}{l}\text { Плотность рассматриваемого газа при стандартных } \\
\text { условиях, кг } / \mathrm{m}^{3} \\
\text { Density of the gas in question under standard condi- } \\
\text { tions, } \mathrm{kg} / \mathrm{m}^{3}\end{array}$ & $\rho_{C T}=0,8580$ \\
\hline $\begin{array}{l}\text { Температура воздуха окружающей среды, К } \\
\text { Ambient temperature }\end{array}$ & $T_{0}=276$ \\
\hline $\begin{array}{l}\text { Значение коэффициента Джоуля-Томсона, К/МПа } \\
\text { Value of the Joule-Thomson coefficient, K/MPa }\end{array}$ & $D i=3,191$ \\
\hline $\begin{array}{l}\text { Расход газа при заданных начальных параметрах, } \\
\text { млн м³/сут } \\
\text { Gas consumption at given initial parameters, } \\
\text { million } \mathrm{m}^{3} / \text { day }\end{array}$ & $Q=6,823$ \\
\hline $\begin{array}{l}\text { Средний коэффициент теплопередачи, Вт/ }\left(\mathrm{m}^{2} \cdot \mathrm{K}\right) \\
\text { Average heat transfer coefficient, } \mathrm{W} /\left(\mathrm{m}^{2} \cdot \mathrm{K}\right)\end{array}$ & $K_{C P}=1,244$ \\
\hline $\begin{array}{l}\text { Относительная плотность газа по воздуху } \\
\text { Relative gas density in air }\end{array}$ & $\Delta=0,62$ \\
\hline $\begin{array}{l}\text { Внутренний диаметр трубопровода, мм } \\
\text { Value of the inner diameter of the pipeline, mm }\end{array}$ & $D_{\theta H}=400$ \\
\hline $\begin{array}{l}\text { Температура газа в трубопроводе, K } \\
\text { Gas temperature in pipeline, } \mathrm{K}\end{array}$ & $T_{C P}=298,932$ \\
\hline $\begin{array}{l}\text { Начальная температура газа в трубопров } \\
\text { Initial gas temperature in pipeline, } \mathrm{K}\end{array}$ & $T_{H}=315$ \\
\hline
\end{tabular}

После проведения расчетов по методике Шухова были получены основные аналитические данные (табл. 3) и отображены графические результаты (рис. 1).

Из графических данных делаем вывод, что при данной эксплуатации температура среды на пятом участке трубопровода ниже, чем температура гидратообразования. Следовательно, этот участок считается началом зоны гидратообразования [20]. До конца протяженности трубопровода (на участках 6-10) во внутренней полости будет происходить аккумулирование гидратов. Для недопущения образования гидратных пробок или скопления гидратов на различных участках, что может привести к снижению общего расхода газа, выхода из строя оборудования или остановки потока, необходимо провести мероприятия по предотвращению гидратообразования.

Таблица 3. Данные по участкам трубопровода

Table 3. Date of pipeline part

\begin{tabular}{|c|c|c|c|c|}
\hline 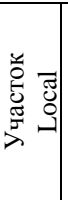 & 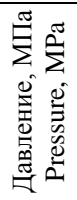 & 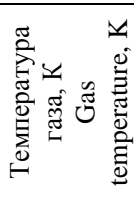 & $\begin{array}{c}\text { Температура } \\
\text { точки росы } \\
\text { газа, K } \\
\text { Gas dew point } \\
\text { temperature, } \mathrm{K}\end{array}$ & $\begin{array}{c}\text { Температура равно- } \\
\text { весного гидратообра- } \\
\text { зования } \\
\text { Temperature of equi- } \\
\text { librium hydrate }\end{array}$ \\
\hline 0,1 & 6,24 & 292,27 & 272,68 & 285,19 \\
\hline 0,2 & 5,97 & 289,97 & 272,07 & 284,83 \\
\hline 0,3 & 5,68 & 287,82 & 271,40 & 284,44 \\
\hline 0,4 & 5,38 & 285,81 & 270,66 & 284,00 \\
\hline 0,5 & 5,06 & 283,92 & 269,83 & 283,51 \\
\hline 0,6 & 4,72 & 282,15 & 268,89 & 282,95 \\
\hline 0,7 & 4,36 & 280,49 & 267,80 & 282,31 \\
\hline 0,8 & 3,96 & 278,94 & 266,50 & 281,53 \\
\hline 0,9 & 3,51 & 277,48 & 264,91 & 280,57 \\
\hline 1 & 3,00 & 276,11 & 262,82 & 279,31 \\
\hline
\end{tabular}

Экспериментальный стенд для борьбы с гидратообразованием

Установка теплового газодинамического воздействия для предотвращения гидратообразования имеет пропускную способность не более $50 \mathrm{~m}^{3} /$ час газа. Схема экспериментального стенда для борьбы с гидратообразованием (льдом в опытных экспериментах) представлена на (рис. 2). Главные задачи, которые необходимо было определить практическим путем с помощью предложенной установки:

- расчет оптимального количества льда для подачи на установку, которое обеспечит максимальную производительность и степень захвата частиц льда;

- определение оптимальных параметров газодинамического потока внутри полости аппарата и конструкции циклона;

- задание оптимальных рабочих параметров процесса для проведения экспериментов [21].

В приемную воронку - 1 подается лед (наиболее близкий по своим химическим свойствам к гидратам элемент) с размерностью частиц до 7 мм. От компрессора, проходя через регулятор давления - 3, подается сжатый воздух с требуемым давлением на эжектор - 2. В результате такого воздействия образуется движение потока газа с частицами льда по транспортной трубе (Ду $=20$ мм), затем двухфазный поток поступает в прямоточный циклон - 5, где отделяются частицы льда из потока и направляются в форбункер с подогревом - 6, где лед (в реальной системе - гидрат) разрушается. Конструктивная особенность данного элемента системы указана на (рис. 3). 


\section{Соотношение температуры газа и температуры гидратообразования} на участке трубопровода

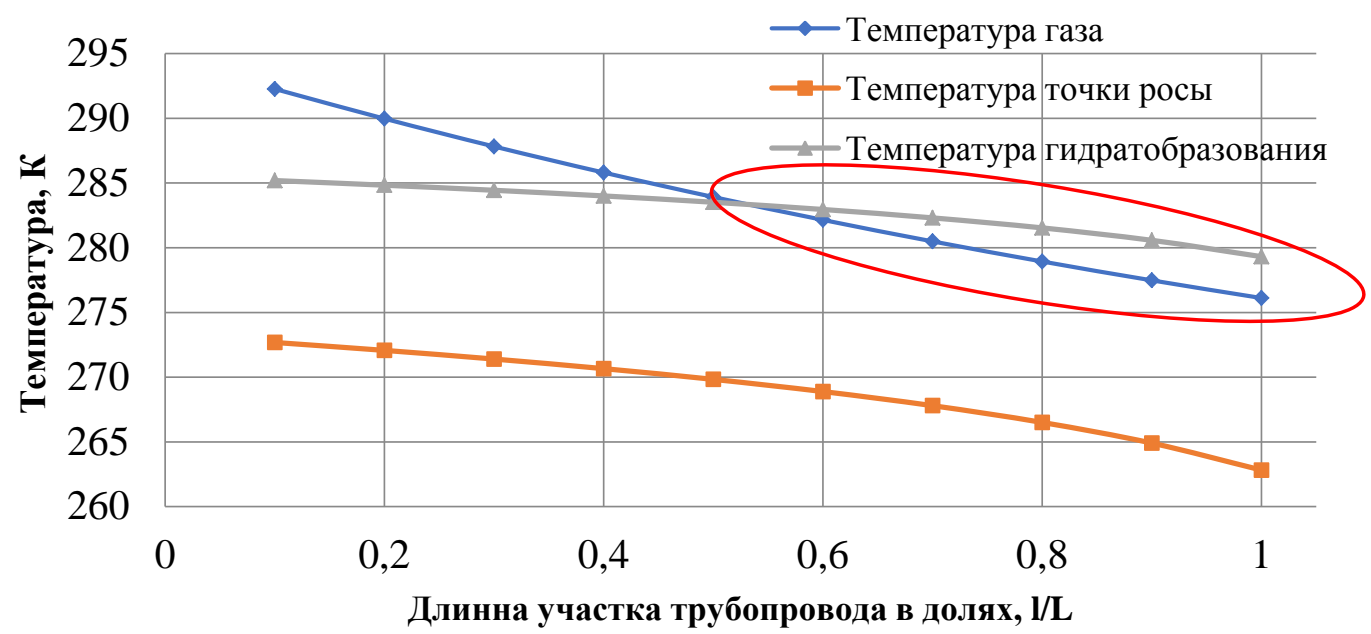

Рис. 1. Результаты расчетов по соотношению температур

Fig. 1. Results of calculations by ratio of temperatures

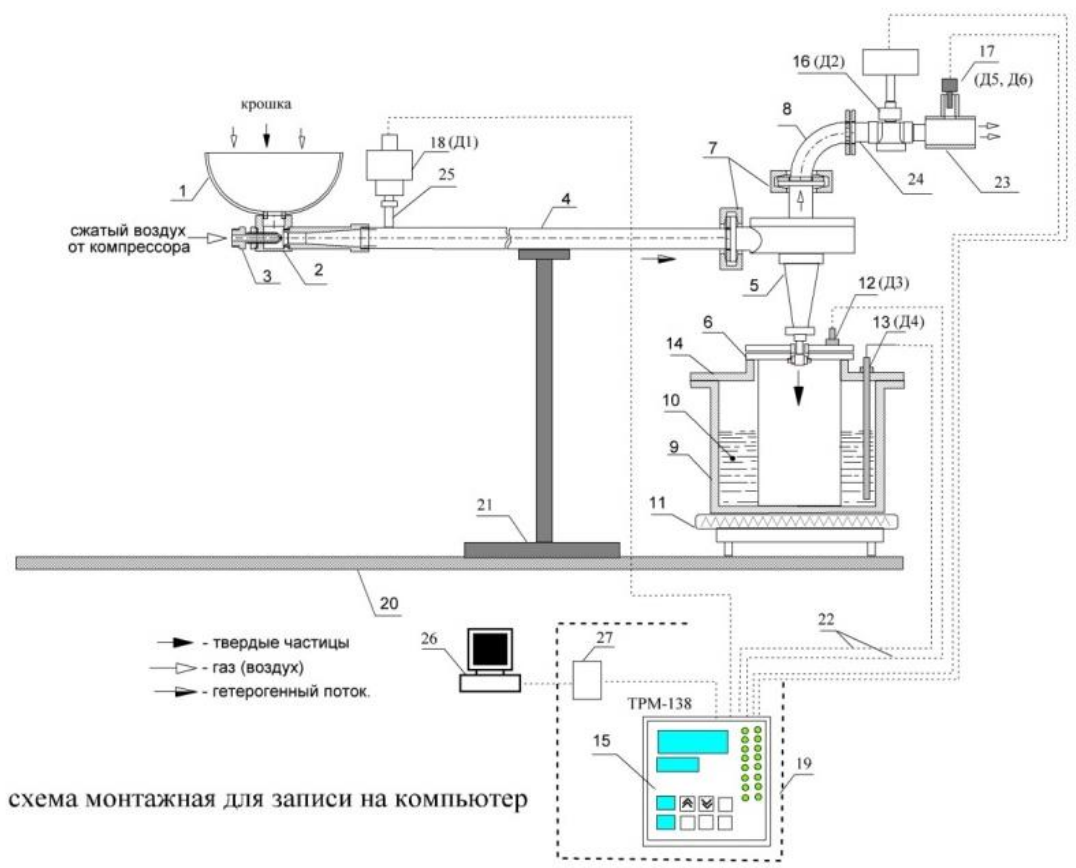

Рис. 2. Схема экспериментальной установки: 1 - воронка приемная; 2 - эжектор с кольцевым соплом; 3 - переходник 27/20 мм; 4 - труба транспортная Dу 20 мм; 5 - ичклон с крышкой; 6- форбункер; 7 - замки; 8 - колено Dу 20 мм; 9 - сосуд с жидкостью; 10 - жидкость (глицерин); 11 - электроплитка 220 B; 12 - датчик температуры газа (ДТС 014-50М.В3.20/1); 13 - датчик температуры жидкости (ДТС 014-50М.В3.20/1); 14 крышка сосуда; 15 - модуль ввода аналоговый МВ 110-224.8A; 16 - электронный датчик разности давлений (ДМ5007А-ДД); 17 - совмещенный датчик влажности и температуры, ДВТ-02 (4-20 мА); 18 - электронный датчик давления (4-20 мА); 19 - шкаф системь управления; 20 - столешница монтажсная; 21 - штатив; 22 - электрические линии связи; 23 - спрямляющая труба с гнездом посадки влагомера; 24 - переходное крепление; 25 -муфта крепления датчика давления; 26 - компьютер; 27 - преобразователь интерфейса RS$485(A C-4)$

Fig. 2. Scheme of the experimental stand: 1 - receiving funnel; 2 - ejector with annular nozzle; 3 - transition 27/20 mm; 4 transport pipe Dy $20 \mathrm{~mm}$; 5 -cyclone with cover; 6 -forbunker; 7 -locks; 8 -transition Dy $20 \mathrm{~mm}$; 9 -vessel with liquid; 10 - liquid (glycerol); 11 - electric stove $220 \mathrm{~V}$; 12 - gas temperature sensor (DTC 014-50M.B3.20/1); 13 liquid temperature sensor (DTC 014-50M.B3.20/1); 14 - vessel cover; 15 - analog input module MB 110-224.8A; 16 - differential pressure sensor (DM 5007A-DD); 17 - combined humidity and temperature sensor, DVT-02 (4-20 mA); 18 - electronic pressure sensor (4-20 mA); 19 - block of control system; 20 - countertop; 21 - tripod; 22 electric communication lines; 23 - straightening tube with hygrometer landing socket; 24 - mount; 25 - coupling of pressure sensor mounts; 26 - computer; 27 - interface convertor $R S-485$ (AC-4) 


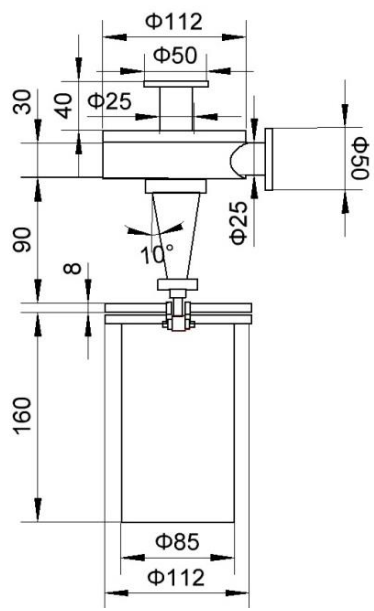

Pис. 3. Система прямоточный циклон - форбункер

Fig. 3. Cyclone-forbunker system

Диаметр сечения сопла для проведения экспериментов: 1-5 мм. Газ продолжает движение дальше, где выходит из системы. Датчики - 12, 13 замеряют температуру среды и температуру теплоносителя для оценки необходимого количества подводимой энергии, датчик - 18 замеряет давление в системе на начальном этапе эксперимента. Датчик - 17 замеряет влагосодержание воздуха, датчик - 18 - общий расход смеси в системе. Все данные в режиме реального времени передаются на компьютер - 26 и в соответствии с методом, описанным в [14], производиться мгновенный расчет. Для обработки информации и интерпретации результатов используется алгоритм, описанный в [22], для получения точных и адаптированных значений термобарических параметров эксперимента.

\section{Расчет параметров циклона}

Из [14] необходимо определить оптимальную скорость течения $\vartheta_{0}=3,5 \mathrm{M} / \mathrm{c}$ для рассматриваемого циклона, после этого по формуле, представленное ниже рассчитать достаточную площадь сечения заданного циклона:

$$
S=\frac{L}{3600 \cdot \vartheta_{0}^{\prime}}
$$

где $L$ - известный объем выбросов, проходящих через циклон, $\mathrm{M}^{3} / \mathrm{ч}$;

$$
S=\frac{15}{3600 \cdot 3,5}=0,0012 \mathrm{~m}^{2} .
$$

Рассчитываем диаметр циклона $(D)$, учитывая, что число циклонов $n=1$, м:

$$
\begin{gathered}
D=1.13 \cdot \sqrt{\frac{S}{n}} ; \\
D=1.13 \cdot \sqrt{\frac{0,0012}{1}}=0,0389 \mathrm{M} .
\end{gathered}
$$

Из [14] выбираем нормируемый диаметр $D_{1}=0,04$ м.

Определяем действительную скорость потока смеси в циклоне:

$$
\vartheta_{0}^{\prime}=\frac{1,27 \cdot L}{3600 \cdot n \cdot D_{1}^{2}}
$$

$$
\vartheta_{0}^{\prime}=\frac{1,27 \cdot 0,0012}{3600 \cdot 1 \cdot 0,04_{1}^{2}}=3,307 \mathrm{~m} / \mathrm{c} .
$$

Действительная скорость движения частиц в циклоне не может отличаться от оптимальной более чем на $15 \%$ :

$$
\begin{gathered}
\Delta=\frac{\vartheta_{0}-\vartheta_{0}^{\prime}}{\vartheta_{0}} \cdot 100 \% ; \\
\Delta=\frac{\vartheta_{0}-\vartheta_{0}^{\prime}}{\vartheta_{0}} \cdot 100 \%=\frac{3,5-3,3}{3,3}=5,5 \% .
\end{gathered}
$$

Для дальнейшего расчета из справочных данных [14] выбираем компоновку циклонов. В соответствии с этими данными определяем коэффициент местного сопротивления $\Delta \varepsilon_{0}=60$. Определяется динамическая вязкость воздуха для рабочих условий $(\mu)$, Па/с:

$$
\mu=1,75 \cdot 10^{-5} \cdot\left(\frac{273+t}{273}\right)^{0,683}
$$

где $t$ - температура окружающей среды в ${ }^{\circ} \mathrm{C}$.

$$
\mu=1,75 \cdot 10^{-5} \cdot\left(\frac{273+20}{273}\right)^{0,683}=1,83 \cdot 10^{-5} \Pi \mathrm{a} / \mathrm{c} .
$$

Также необходимо рассчитать аэродинамическое сопротивление прямоточного циклона $\left(\Delta P_{ц}\right)$, Па, по формуле:

$$
\Delta P_{\text {Ц }}=\xi_{\longleftrightarrow} \cdot\left(\frac{\rho_{\mathrm{B}} \cdot \vartheta_{0}^{\prime 2}}{2}\right),
$$

где $\rho_{\mathrm{B}}=0,646-$ сопротивление потока воздуха; $\xi_{ц}-$ коэффициент местного сопротивления, который отнесен к скорости $\vartheta_{0}^{\prime}$ и определен с учетом поправочных коэффициентов по формуле:

$$
\xi_{\text {ц }}=k_{1} \cdot k_{2} \cdot \xi_{0}+\Delta \xi_{0},
$$

где $\Delta \xi_{0}-$ коэффициент, зависящий от вариации компоновки циклона по справочным данным [14]; $k_{1}-$ коэффициент, зависящий непосредственно от диаметра проходного отверстия циклона [14]; $k_{2}$ - справочный коэффициент из [14] для учета запыленности воздуха; $\xi_{0}$ - коэффициент местного сопротивления из [14]:

$$
\begin{aligned}
\xi_{ц} & =0,94 \cdot 0,95 \cdot 5,25+60=64,68 ; \\
\Delta P_{ц} & =64,68 \cdot\left(\frac{0,646 \cdot 3,3^{2}}{2}\right)=255,95 \text { Па. }
\end{aligned}
$$

По графику фракционной эффективности очистки из [14] определяем размер частиц $d_{50} d_{50}$ с эффективностью улавливания 97,8 \% для выбранного типа циклона с $D_{1}$. Для определения эффективности циклона других размеров и скорости движения воздуха, его вязкости и плотности вычисляют новое значение $d_{50}^{\prime}$ мкм по формуле:

$$
d_{50}^{\prime}=548,5 \cdot d_{50} \cdot \sqrt{\frac{D_{1} \cdot \mu \cdot \vartheta_{0}}{P n \cdot \vartheta_{0}^{\prime}}},
$$

где $P n-$ плотность частиц $\left(P n=10 \mathrm{a} 00\right.$ кг $\left./ \mathrm{m}^{3}\right)$,

$$
d_{50}^{\prime}=548,5 \cdot d_{50} \cdot \sqrt{\frac{0,04 \cdot 1,83 \cdot 10^{-5} \cdot 3,5}{1000 \cdot 3,3}}=0,00413 \mathrm{~m} .
$$

Основные размеры циклона должны быть следующими:

- выходное отверстие циклона:

$$
D_{\text {вых }}=0,59 \cdot D_{1}=0,59 \cdot 0,04=0,025 \mathrm{~m} ;
$$


- размеры входного патрубка $a, b$ :

$$
\begin{gathered}
a=1,11 \cdot D_{1}=1,11 \cdot 0,04=0,044 \mathrm{~m} ; \\
b=0,26 \cdot D_{1}=0,26 \cdot 0,04=0,01 \mathrm{M} ;
\end{gathered}
$$

- общая высота циклона:

$$
H=3,1 \cdot D_{1}=3,1 \cdot 0,04=0,124 \mathrm{M} .
$$

\section{Проведение экспериментов}

Эксперимент 1 - Определение степени отделения

Эксперимент проводился при следующих условиях: постоянное значение давление подачи воздуха $P=2$ атм., масса льда $m=150$ г, размерность частиц льда 2-4 мм, диаметр сопла $d=2$ мм, постоянный коэффициент эжекции. Температура воздуха в форбункере и температура теплоносителя поддерживаются изменением мощности нагревателя и остаются неизменными на протяжении всего эксперимента. Задача состояла в том, чтобы найти массу льда, который не отделился из потока смеси. Время проведения эксперимента от 5 до 15 секунд.

\section{Эксперимент 2 - Определение степени отделения}

Эксперимент проводился при следующих условиях: постоянное значение давление подачи воздуха $P=3$ атм., масса льда $m=150$ г, размерность частиц льда 2-4 мм, диаметр сопла $d=3$ мм, постоянный коэффициент эжекции. Температура воздуха в форбункере и температура теплоносителя поддерживаются изменением мощности нагревателя и остаются неиз- менными на протяжении всего эксперимента. Задача состояла в том, чтобы найти массу льда, который не отделился из потока смеси. Время проведения эксперимента от 3,5 до 14 секунд.

\section{Эксперимент 3 - Определение степени отделения}

Эксперимент проводился при следующих условиях: постоянное значение давление подачи воздуха $P=4$ атм., масса льда $m=150$ г, дисперсность льда 2-4 мм, диаметр сопла $d=4$ мм, постоянный коэффициент эжекции. Температура воздуха в форбункере и температура теплоносителя поддерживаются изменением мощности нагревателя и остаются неизменными на протяжении всего эксперимента. Задача состояла в том, чтобы найти массу льда, который не отделился из потока смеси.

В первых трех экспериментах физическая задача состоит в том, чтобы определить зависимость степени отделения твердых частиц от начальной их концентрации и от объемного расхода газа. Расход газа изменяется входным давлением и диаметром сопла, концентрация льда в исходном потоке изменяется временем введения частиц в систему: $m$ льда=150 m/t введения (г/с). Температура теплоносителя постоянна. Время проведения эксперимента от 2,7 до 11 секунд.

На рис. 4 приведены результаты экспериментов 1-3. Представлен общий график распределения зависимости степени отделения частиц льда от их начальной концентрации и от динамики потока в сравнении.

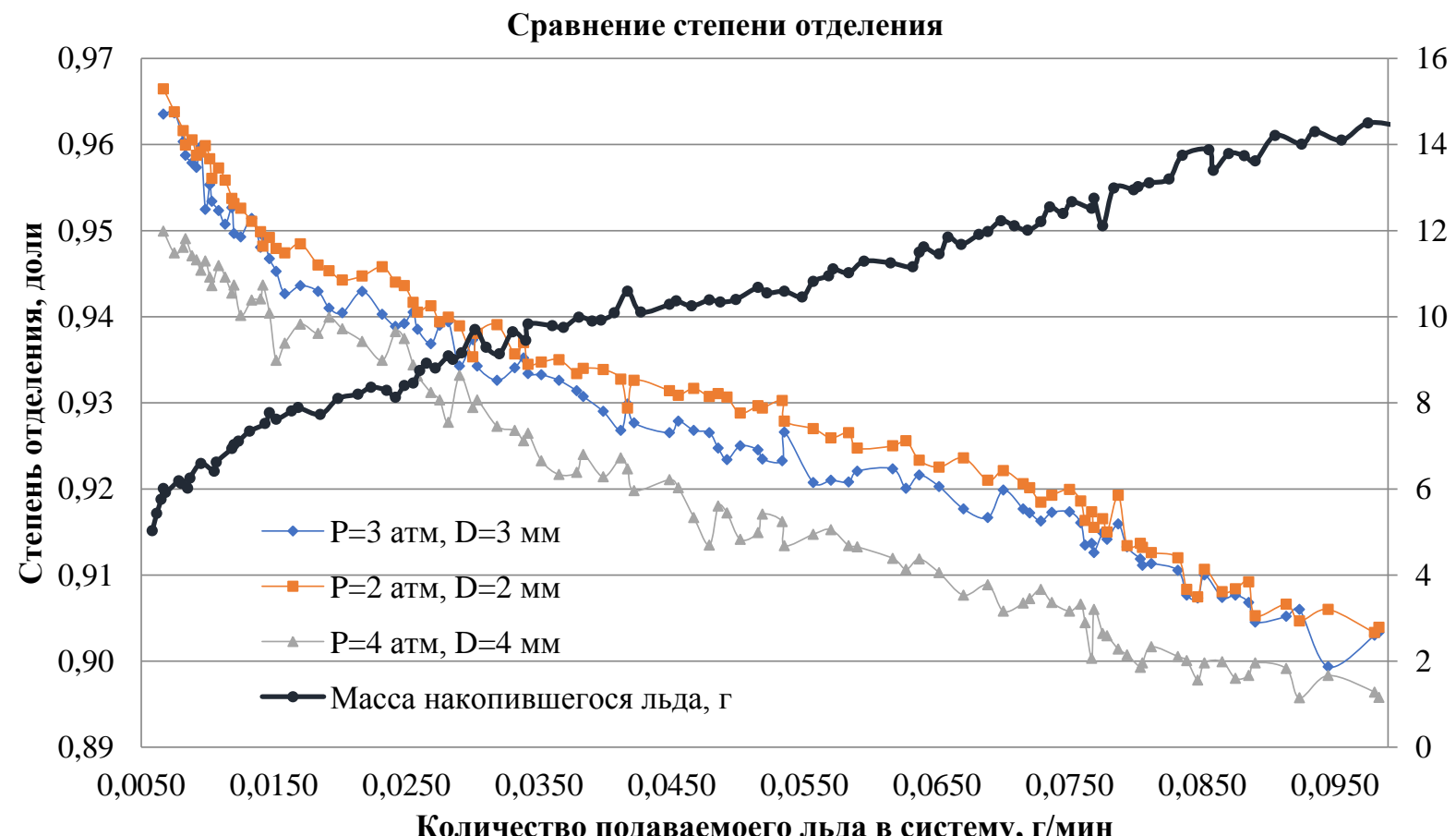

Рис. 4. Обобщенные результаты экспериментов 1-3

Fig. 4. Generalized results of the experiments 1-3

Из рис. 4 видно, что чем выше динамика потока и чем выше исходная концентрация, тем хуже происходит отделение частиц. Для проведения эксперимента по тепловому воздействию воспользуемся моделью № 1, где произошло лучшее отделение льда из потока смеси. Экспериментально определим время восста- 
новления температуры теплоносителя до его начальной температуры. Рассмотрим два температурных режима: $\mathrm{t}_{\text {начальное }}=60{ }^{\circ} \mathrm{C}$ и $\mathrm{t}_{\text {начальное }}=80^{\circ} \mathrm{C}$.

Эксперимент 4 - Определение времени восстановления температуры теплоносителя

Эксперимент проводился при следующих условиях: постоянное значение давление подачи воздуха $P=2$ атм., масса льда $m=150$ г, дисперсность льда 2-4 мм, диаметр сопла $d=2$ мм, постоянный коэффициент эжекции. Начальная температура теплоносителя составляет $60{ }^{\circ} \mathrm{C}$. Время проведения эксперимента от 3 до 10 секунд.
Эксперимент 5 - Определение времени восстановления температуры теплоносителя

Эксперимент проводился при следующих условиях: постоянное значение давление подачи воздуха $P=2$ атм., масса льда $m=150$ г, дисперсность льда 2-4 мм, диаметр сопла $d=2$ мм, постоянный коэффициент эжекции. Начальная температура теплоносителя составляет $80{ }^{\circ} \mathrm{C}$. Время проведения эксперимента от 3 до 10 секунд.

На рис. 5, 6 графически отображены результаты эксперимента 4 и 5. На рис. 7, 8 отображены скачки температуры при попадании в систему льда для эксперимента 4 и 5 соответственно, при этом температура теплоносителя не поддерживается.

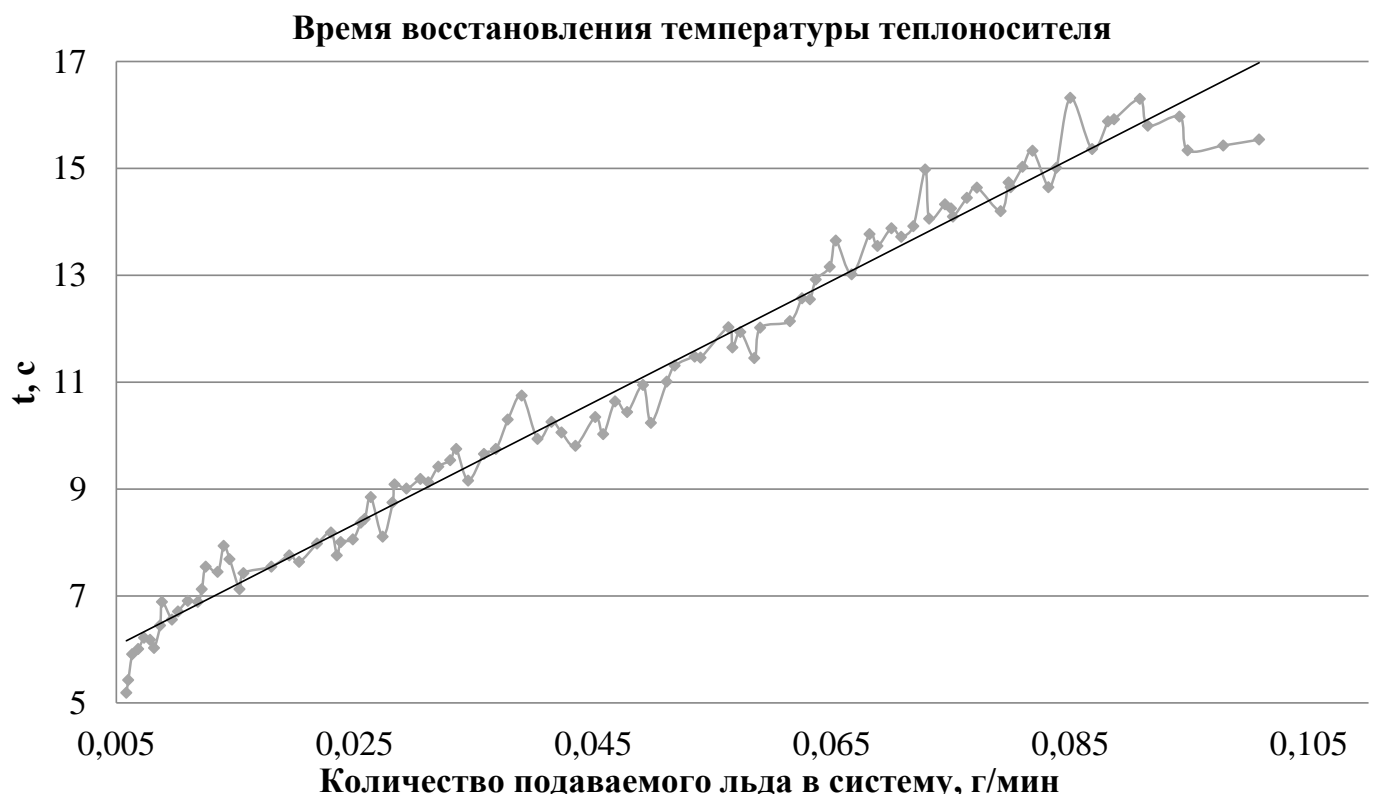

Рис. 5. Зависимость времени восстановления температуры от начальной концентрации льда $\left(t_{\text {начальное }}=60^{\circ} \mathrm{C}\right)$ Fig. 5. Dependence of the temperature recovery time on the initial ice concentration $\left(t=60^{\circ} \mathrm{C}\right)$

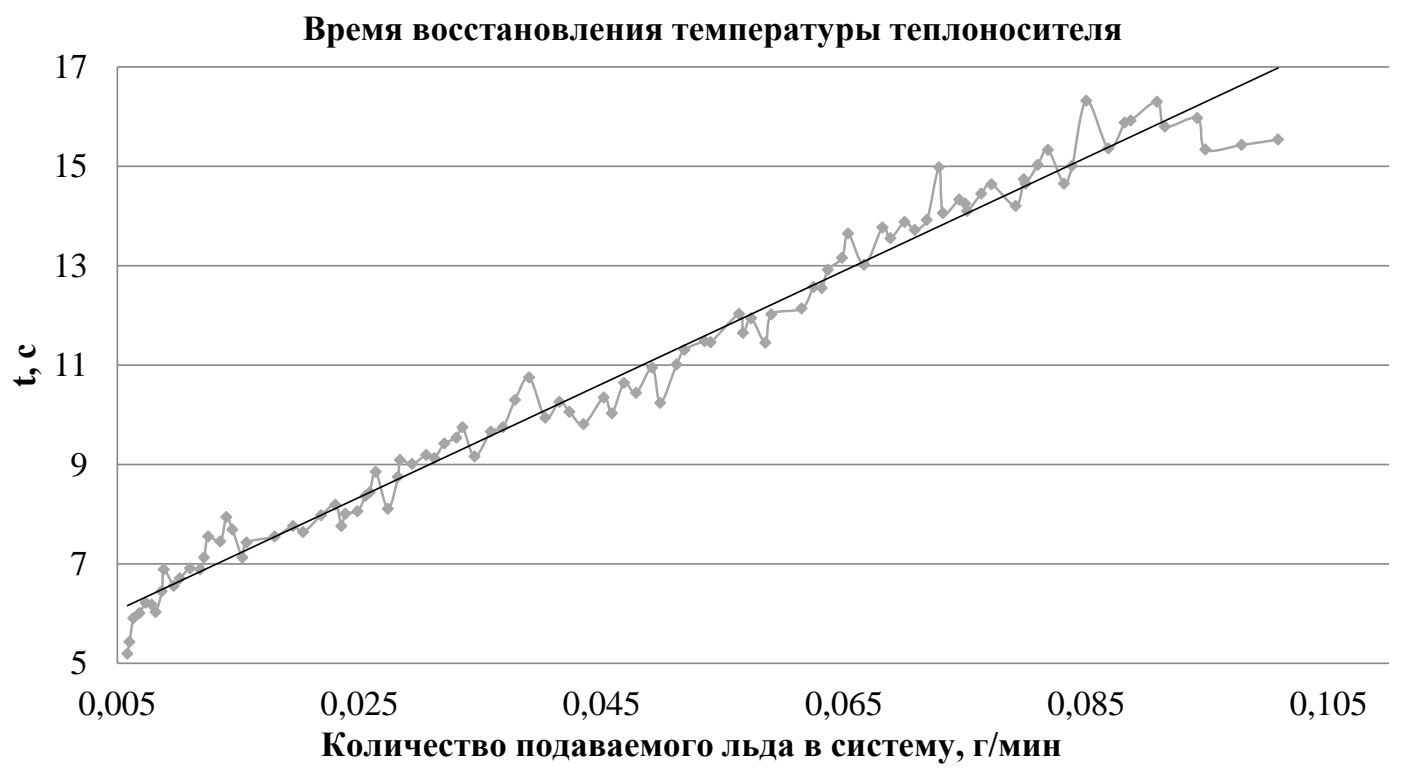

Рис. 6. Зависимость времени восстановления температуры от начальной концентрации льда $\left(t_{\text {начальное }}=80^{\circ} \mathrm{C}\right)$

Fig. 6. Dependence of the temperature recovery time on the initial ice concentration $\left(t=80^{\circ} \mathrm{C}\right)$ 


\section{Температура теплоносителя после попадания льда в систему}

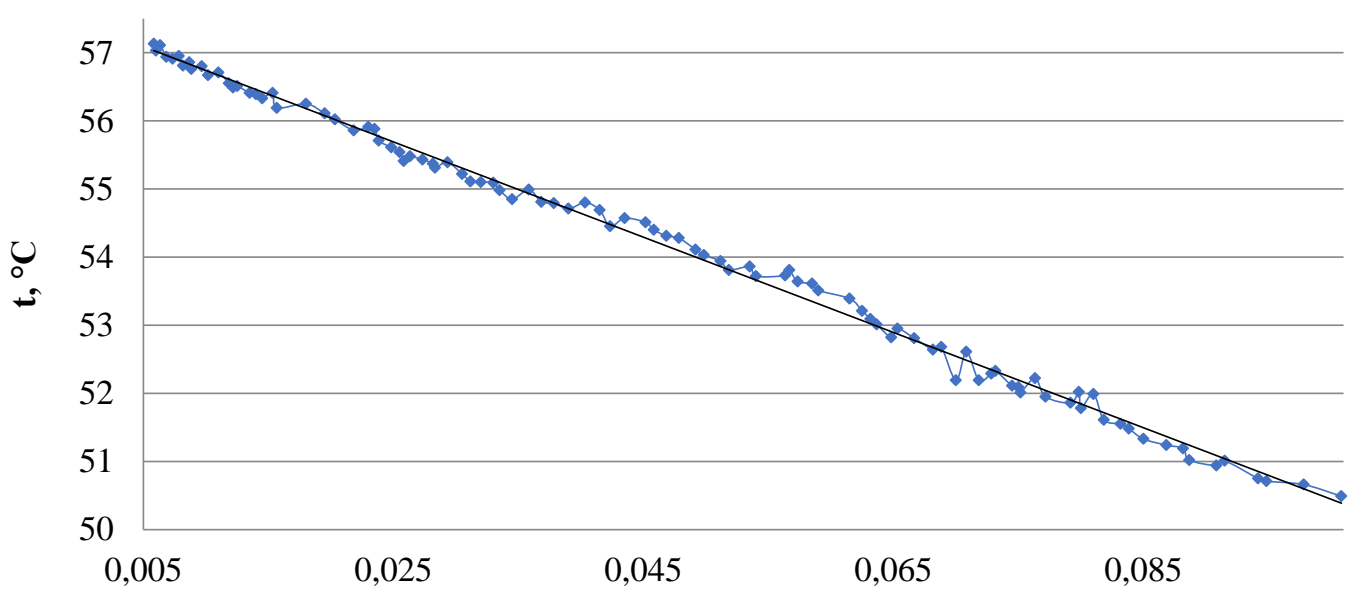

Количество подаваемого льда в систему, г/мин

Рис. 7. Зависимость скачка температуры при попадании льда в форбункер от начальной концентрации льда $\left(t_{\text {начальное }}=60^{\circ} \mathrm{C}\right.$ )

Fig. 7. Dependence of temperatyre jump after ice enter in forbunker on initial ice concentration $\left(t=60^{\circ} \mathrm{C}\right)$

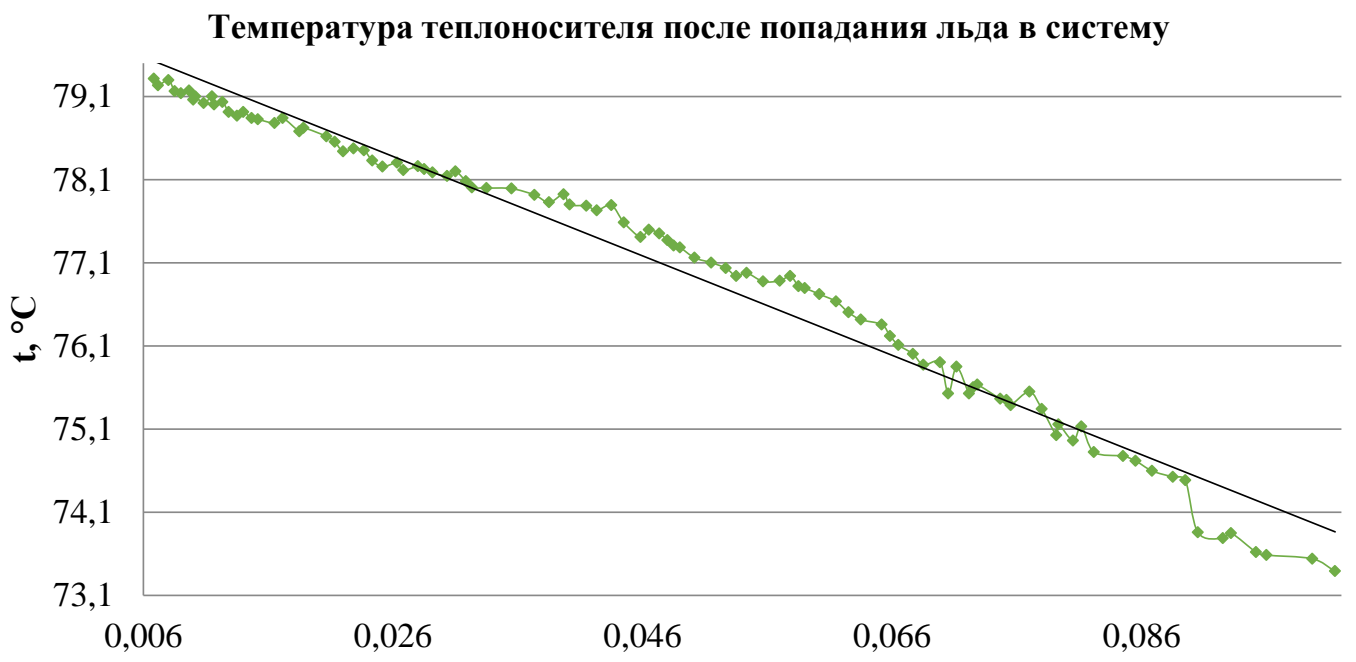

Количество подаваемое льда в систему, г/мин

Рис. 8. Зависимость скачка температуры при попадании льда в форбункер от начальной кониентрации льда $\left(t_{\text {начальное }}=80^{\circ} \mathrm{C}\right)$

Fig. 8. Dependence of temperatyre jump after ice enter in forbunker on initial ice concentration $\left(t=80^{\circ} \mathrm{C}\right)$

Из графиков, представленных выше, видно, что чем выше температура, тем ниже начальный скачок температуры и меньше время восстановления. Самый оптимальный эксперимент при температуре теплоносителя $\mathrm{t}_{\text {начальное }}=80^{\circ} \mathrm{C}$ и концентрации порядка 0,5 г/c.

Экспериментальный стенд разработан при поддержке грантовой программы нефтегазовой компании British Petroleum.

\section{Заключение}

Создан экспериментальный стенд для борьбы с гидратообразованием, который показал эффектив- ность практического применения аэромеханического метода воздействия для предотвращения гидратообразования. В результате проведенных экспериментов получены оптимальные термобарические параметры для проведения дальнейших исследований и выдачи рекомендаций для промышленного образца модульной вставки борьбы с гидратообразованием. Модернизирован и автоматизирован экспериментальный стенд, изучены основные зависимости и степени их влияния на технологические параметры процесса разделения и разрушения гидратов. 


\section{СПИСОК ЛИТЕРАТУРЫ}

1. Fortin G., Ilinca A., Brandi V. A new roughness computation method and geometric accretion model for airfoil icing // Journal of Aircraft. - 2016. - V. 41. - № 1. - P. 119-127.

2. Бешенцева С.А. Анализ методов предупреждения гидратообразования в трубопроводах // Вестник кибернетики. - 2017. № 11. - С. 40-44.

3. Галимзянов М.Н. Математическое моделирование образования газовых гидратов // Вестник Башкирского университета. 2018. - Т. 15. - № 3. - С. 570-574.

4. Попов Д.Н., Варфоломеева О.И., Хворенков Д.А. Особенности неизотермических течений жидкости с переменными реологическими свойствами в каналах с местными сопротивлениями // Вестник Ижевского государственного технического университета. - 2016. - № 1 (57). - С. 146-149.

5. Комисссаров Ю.А., Гордеев Л.С., Вент Д.П. Процессы и аппараты химической технологии. - М.: Химия, 2018. - 250 c.

6. Исследование гидродинамических и термодинамических процессов высоконапорного многофазного вихревого течения жидкости / Ю.М. Ахметов, Р.Р. Калимуллин, Р.Ф. Хакимов, С.Ю. Константинов, Д.В. Целищев // Вестник Уфимского государственного авиационного технического университета. 2016. - T. 16. - № 2. - C. 163-168.

7. Abgrall R., Saurel R. Discrete equations for physical and numerical compressible multiphase mixtures // Journal of Computational Physics. - 2016. - V. 186. - P. 361-396.

8. Application of numerical signal differentiation methods to determine statio-narity of A process / A.V. Maystrenko, A.A. Svetlakov, T.V. Gandzha, V.V. Dmitriev, N.V. Aksenova // Petroleum and Coal. - 2017. - V. 59. - Iss. 3. - P. 311-318.

9. Saurel R., Petitpas F., Berry R.A. Simple and efficient relaxation methods for interfaces separating compressible fluids, cavitating flows and shocks in multiphase mixtures // Journal of Computational Physics. - 2017. - V. 228. - Iss. 5. - P. 1678-1712.

10. Вараксин А.В. Гидрогазодинамика и теплофизика двухфазных потоков: проблемы и достижения // Теплофизика высоких температур. - 2017. - № 3. - С. 421-455.

11. Волков П.В., Большунов А.В. Исследование и комплексное применение гидроаэромеханических методов для предотвращения гидратообразования при подготовке газа // Научный журнал «Успехи современного естествознания». - 2017. № 9 . - С. 52-56.
12. Методы и средства предотвращения гидратообразования на объектах газодобычи / М.Ю. Прахова, А.Н. Краснов, Е.А. Хорошавина, Э.А. Шаловников // Нефтегазовое дело. - 2016. № 1. - C. 101-118.

13. Запорожец Е.П., Шостак Н.А. Гидраты. - Краснодар: ИД «Юг», 2017. - 460 c.

14. Запорожец Е.П., Антониади Д.Г., Зиберт Г.К. Регулярные процессы и оборудование в технологиях сбора, подготовки и переработки нефтяных и природных газов: учебное пособие. Краснодар: ИД «Юг», 2017. - 620 с.

15. Шагаов В.Ш., Тазетдинов Б.И. К теории разложения метастабильного газогидрата // Теоретические основы химической технологии. - 2017. - Т. 47. - № 4. - С. 454-463.

16. Derevich I.V. The hydrodynamics and heat transfer and mass transfer of particles under conditions of turbulent flow of gas suspension in a pipe and in an axisymmetric jet // Journal of High Temperature. - 2016. - V. 40. - № 1. - Р. 78-91.

17. Ширшова А.В., Данько М.Ю. Рост и диссоциация газогидратов в водонефтяных эмульсиях // Известия вузов. Сер. Нефть и газ. - 2017. - № 5. - С. 95-101.

18. Gavrilyuk S.L., Saurel R. Mathematical and numerical modeling of two phase compressible flows with micro inertia // Journal of Computational Physics. - 2016. - V. 175. - Iss. 1. - P. 326-360.

19. Naseer M., Brandstatter W. Hydrate formation in natural gas pipelines // WIT Transactions on Engineering Sciences. - 2017. V. 70. - P. 261-270.

20. An algorithm to improve the speed and accuracy of analysis of chemical process systems operation / V.V. Dmitriev, T.V. Gandzha, I.M. Dolganov, N.V. Aksenova // Petroleum and Coal. - 2017. - V. 59. - Iss. 3. - P. 429-441.

21. Волков П.В. Экспериментальное исследование аэромеханического метода для предотвращения гидратообразования при разработке газовых месторождений и установок комплексной подготовки газа // Нефть и газ 2019: Сборник трудов 73-й международной молодежной научной конференции. - М., 2019. - T. 1. - C. 78-83.

22. Lovick J., Angeli P. Experimental studies on the dual continuous flow pattern in oil-water flows // International Journal of Multiphase Flow. - 2004. - V. 30. - P. 139-157.

Поступила 17.06.2020 2.

\section{Информация об авторах}

Зятиков П.Н., доктор технических наук, профессор отделения нефтегазового дела Инженерной школы природных ресурсов Национального исследовательского Томского политехнического университета.

Волков П.В., младший научный сотрудник АО ТомскНИПИнефть; аспирант отделения нефтегазового дела Инженерной школы природных ресурсов Национального исследовательского Томского политехнического университета.

Большунов A.B., аспирант отделения нефтегазового дела Инженерной школы природных ресурсов Национального исследовательского Томского политехнического университета. 
UDC 622.279 .8

\title{
EXPERIMENTAL RESEARCH OF HEAT GAS-DYNAMICS METHOD FOR PREVENTING HYDRATE FORMATION IN GAS PIPELINES USING CYCLONES
}

\author{
Pavel N. Zyatikov 1 , \\ zpavel@tpu.ru
}

Pavel V. Volkov2,1,

tpuipr@mail.ru

\author{
Artem V. Bolshunov', \\ tpuiprgrnm@mail.ru \\ 1 National Research Tomsk Polytechnic University, \\ 30, Lenin avenue, Tomsk, 634050, Russia. \\ 2 TomskNIPIneft, \\ 72, Mira avenue, Tomsk, 634027, Russia
}

The relevance of the study is caused by the need to reduce the amount of gas hydrates in the mixture stream or their complete destruction. In the oil and gas industry, gas hydrates are a negative factor, since they greatly complicate production, transportation and preparation of hydrocarbon raw materials. Under certain thermobaric conditions, hydrates precipitate and accumulate on the walls of vessels, pipelines and in the cavities of the valves, which leads to their wear and increase of hydraulic resistance, and, consequently, to increased energy costs. Existing methods to combat hydrate formation are very dangerous (using methanol to destruct hydrates), very expensive (using hydrate inhibitors) or energy consuming and not effective (heating the pipeline with a specialized cable). Also, accumulation of hydrates can adversely affect metering and metering devices.

The main aim of the research is the experimental study of removing ice from a gas stream and its destruction in a specialized installation. Object: autonomous modular installation for combating hydrate treatment.

Methods: theoretical methods to justify the patterns of heat and mass transfer, a calculation-analytical method for determining the amount of energy, practical methods for conducting experiments.

Results. The laboratory stand was built on the basis of National Research Tomsk Polytechnic University; experiments were conducted for various gas flows with different thermobaric conditions; the main dependences of the quality of separation of ice from the mixture were analyzed; the practical significance of the development was proved; and recommendations were made for creating a prototype. A model for calculating the main structural parameters of a cyclone and a working cavity is considered. A methodology for conducting experiments and techniques for selecting optimal thermobaric parameters is presented.

\section{Key words:}

Hydrate formation, cyclone, gasodynamic method, heat and mass transfer, dynamic.

\section{REFERENCES}

1. Fortin G. A new roughness computation method and geometric accretion model for airfoil icing. Journal of Aircraft, 2016, vol. 41, no. 1, pp. 119-127.

2. Beshentseva S.A. Analysis of methods for preventing hydrate formation in pipelines. Herald of cybernetics, 2017, no. 11, pp. 40-44. In Rus.

3. Galimzyanov M.N. Mathematical modeling of the formation of gas hydrates. Herald of Bashkir University, 2018, vol. 15, no. 3, pp. 570-574. In Rus.

4. Popov D.N., Varfolomeeva O.I., Khvorenkov D.A. Features of nonisothermal fluid flows with variable rheological properties in channels with local resistances. Herald of the Izhevsk State Technical University, 2016, no. 1 (57), pp. 146-149. In Rus.

5. Komissssarov Yu.A., Gordeev L.S., Vent D.P. Processy i apparaty khimicheskoy tekhnologii [Processes and devices of chemical technology]. Moscow, Khimiya Publ., 2018. 250 p.

6. Akhmetov Yu.M., Kalimullin R.R., Khakimov R.F., Konstantinov S.Yu., Tselishchev D.V. Investigation of the hydrodynamic and thermodynamic processes of high-pressure multiphase vortex fluid flow. Herald of Ufa State Aviation Technical University, 2016, vol. 16, no. 2, pp.163-168. In Rus.

7. Abgrall R., Saurel R. Discrete equations for physical and numerical compressible multiphase mixtures. Journal of Computational Physics, 2016, vol. 186, pp. 361-396.

8. Maystrenko A.V., Svetlakov A.A., Gandzha T.V., Dmitriev V.V., Aksenova N.V. Application of numerical signal differentiation methods to determine statio-narity of a process. Petroleum and Coal, 2017, vol. 59, Iss. 3, pp. 311-318.
9. Saurel R., Petitpas F., Berry R.A. Simple and efficient relaxation methods for interfaces separating compressible fluids, cavitating flows and shocks in multiphase mixtures. Journal of Computational Physics, 2017, vol. 228, Iss. 5, pp. 1678-1712.

10. Varaksin A.V. Hydrogasdynamics and thermophysics of twophase flows: problems and achievements. Thermophysics of high temperatures, 2017, no. 3, pp. 421-455. In Rus.

11. Volkov P.V., Bolshunov A.V. Research and integrated application of hydroaeromechanical methods to prevent hydrate formation during gas preparation. Successes in modern natural sciences, 2017, no. 9, pp. 52-56. In Rus.

12. Prakhova M.Yu., Krasnov A.N., Khoroshavina E.A., Shalovnikov E.A. Methods and means of preventing hydrate formation at gas production facilities. Oil and Gas Business, 2016, no. 1, pp. 101-118. In Rus.

13. Zaporozhets E.P. Gidraty [Hydrates]. Krasnodar, Yug Publ. House, 2017. $460 \mathrm{p}$

14. Zaporozhets E.P. Regularnye processi i oborudovanie v technologiakh sbora, podgotovki $i$ pererabotki [Regular processes and equipment in the technology of collection, preparation and processing of oil and natural gases]. Krasnodar, Yug Publ. House, 2017. $620 \mathrm{p}$.

15. Shagov V.Sh. To the theory of decomposition of metastable gas hydrate. Theoretical foundations of chemical technology, 2017, vol. 47, no. 4, pp. 454-463. In Rus.

16. Derevich I.V. The hydrodynamics and heat transfer and mass transfer of particles under conditions of turbulent flow of gas suspension in a pipe and in an axisymmetric jet. Journal of High Temperature, 2016, vol. 40, no. 1, pp. 78-91. 
17. Shirshova A.V. Growth and dissociation of gas hydrates in oilwater emulsions. University News. Ser. Oil and gas, 2017, no. 5, pp. 95-101. In Rus.

18. Gavrilyuk S.L., Saurel R. Mathematical and numerical modeling of two phase compressible flows with micro inertia. Journal of Computational Physics, 2016, vol. 175, Iss. 1, pp. 326-360.

19. Naseer M., Brandstatter W. Hydrate formation in natural gas pipelines. WIT Transactions on Engineering Sciences, 2017, vol. 70, pp. 261-270.

20. Dmitriev V.V., Gandzha T.V., Dolganov I.M., Aksenova N.V. An algorithm to improve the speed and accuracy of analysis of chemical process systems operation. Petroleum and Coal, 2017, vol. 59 Iss. 3, pp. 429-441.
21. Volkov P.V. Experementalnoe issledovanie aeromekhanicheskogo metoda dlya predotvrashcheniya gidratoobrazovaniya pri razrabotke gazovykh mestorozhdeniy i ustanovok kompleksnoy podgotovki gaza [An experimental study of the aeromechanical method to prevent hydrate formation during the development of gas fields and integrated gas treatment plants]. Neft i gaz 2019. Sbornik trudov 73 mezhdunarodnoy molodezhnoy nauchnoy konferentsii [Proc. of the $73^{\text {rd }}$ international youth scientific conference. Oil and Gas 2019]. Moscow, 2019, vol. 1, pp. 78-83.

22. Lovick J., Angeli P. Experimental studies on the dual continuous flow pattern in oil-water flows. International Journal of Multiphase Flow, 2004, vol. 30, pp. 139-157.

Received: 17 June 2020.

\section{Information about the authors}

Pavel N. Zyatikov, Dr. Sc., professor, National Research Tomsk Polytechnic University.

Pavel V. Volkov, junior researcher, TomskNIPIneft; aspirant, National Research Tomsk Polytechnic University. Artem V. Bolshunov, postgraduate student, National Research Tomsk Polytechnic University. 